\title{
Consumer Preferences for Bundles and Bundle Components with Odd and Even Price Endings
}

\author{
Bernhard Baumgartner and Anjulie Hähnchen
}

\begin{abstract}
Marketing literature suggests that price endings can have an impact on consumers' evaluations of the product as well as his/her intention to buy. Retailers often use odd prices, e.g. prices ending in '9'. While also bundles of products are regularly offered with odd prices, literature on the application of odd and even prices in bundles is nearly non-existent. In this research, we investigate the impact of odd and even priced bundle components as well as odd and even total prices on consumer preferences.

Choice-based conjoint analysis is applied to investigate preferences. Using a hierarchical Bayesian approach, we allow for consumer heterogeneity. Individualspecific variables (price- and quality consciousness) are included as concomitant variables. Members of a national online panel completed an online questionnaire, including 11 choice sets. In each choice set, the respondents chose between purchasing a desk chair, a trolley, a bundle of these products or none of these options. The products and the bundle were presented with varying prices ending in odd or even numbers.

We find only weak indication for direct effects of an odd component price on the choice probability of the respective component. The preference for the bundle increases if both components are labelled with even prices instead of using odd component prices. This result suggests that consumers use even prices as an indicator of quality and therefore prefer bundles consisting of even priced components. The preference for the bundle does not only depend on the price ending of the components, but also on the last digit of the bundle price. The bundle choice probability increases with an odd bundle price as compared to an even bundle price. This outcome appears plausible because we do not expect a quality image effect for the bundle price. Therefore, the odd total price causes level effects or price image effects. As a consequence, the probability of buying the bundle is highest if both components are labelled with even prices, whereas the total bundle price has an odd price ending. We did not find evidence for moderating effects of price- or quality consciousness.
\end{abstract}

B. Baumgartner $(\bowtie) \bullet$ A. Hähnchen

University of Osnabrueck, Osnabrück, Germany

e-mail: bebaumga@uos.de; ahaehnch@uos.de

L. Petruzzellis, R.S. Winer (eds.), Rediscovering the Essentiality of Marketing,

Developments in Marketing Science: Proceedings of the Academy of Marketing

Science, DOI 10.1007/978-3-319-29877-1_114 